\title{
Understanding Rook Endgames
}

\author{
Article
}

Accepted Version

Haworth, G. (2017) Understanding Rook Endgames. ICGA Journal, 39 (2). pp. 145-150. ISSN 1389-6911 Available at https://centaur.reading.ac.uk/65694/

It is advisable to refer to the publisher's version if you intend to cite from the work. See Guidance on citing.

Publisher: The International Computer Games Association

All outputs in CentAUR are protected by Intellectual Property Rights law, including copyright law. Copyright and IPR is retained by the creators or other copyright holders. Terms and conditions for use of this material are defined in the End User Agreement.

\section{www.reading.ac.uk/centaur}

\section{CentAUR}

Central Archive at the University of Reading

Reading's research outputs online 


\section{Understanding Rook Endgames}

Guy Haworth

Reading, $U K^{1}$

Understanding Rook Endgames (Müller and Konoval, 2016) or URE is the latest contribution of many to endgame lore by Gambit Publications, and is the first such work totally checked by 7-man and sub-7-man 'EGT' endgame table verification. It is available not only in paperback but on Apple/Android devices courtesy of the Gambit Chess Studio app, a particularly useful tool here for the mobile reader following the analysis.

Table 1

maxDTx data for the featured sub-8-man rook endgames. ${ }^{2}$

\begin{tabular}{|c|c|c|c|c|c|c|c|c|c|c|c|c|c|}
\hline \multirow{3}{*}{\multicolumn{2}{|c|}{ \# \#m }} & \multirow{3}{*}{\multicolumn{2}{|c|}{ w-b Endgame }} & \multicolumn{10}{|c|}{ maxDTx in winner's moves } \\
\hline & & & & \multicolumn{5}{|c|}{ " } & \multicolumn{5}{|c|}{$0-1$} \\
\hline & & & & \multicolumn{5}{|c|}{ DTC DTM DTZ DTZ ${ }_{50}$ DTZ $_{50}^{\prime}-\mathrm{fw}$} & \multicolumn{5}{|c|}{ DTC DTM DTZ DTZ ${ }_{50}$ DTZ $_{50}^{\prime}-\mathrm{fw}$} \\
\hline 1 & 4 & $2-2$ & KRKP & 16 & 32 & 13 & 13 & - & 11 & 43 & 10 & 10 & - \\
\hline 2 & 5 & $3-2$ & KRPKR & 60 & 74 & 35 & 35 & - & 7 & 33 & 7 & 7 & - \\
\hline 3 & 6 & $4-2$ & KRPPKR & 86 & 95 & $?$ & 45 & - & 8 & 42 & $?$ & 8 & - \\
\hline 4 & 6 & $3-3$ & KRPKRP & 41 & 116 & $?$ & 37 & 11 & 41 & 116 & $?$ & 37 & 11 \\
\hline 5 & 7 & $4-3$ & KRPPKRP & 79 & 217 & $?$ & $?$ & $?$ & 41 & 144 & $?$ & $?$ & $?$ \\
\hline 6 & 7 & $4-3$ & KQRPKQR & 82 & 137 & $?$ & $?$ & $?$ & 94 & 145 & $?$ & $?$ & $?$ \\
\hline 7 & 7 & 4-3 & KRRPKRR & 135 & 295 & $?$ & $?$ & $?$ & 27 & 82 & $?$ & $?$ & $?$ \\
\hline
\end{tabular}

The authors scarcely need any introduction. Karsten Müller is the major and most generous purveyor of endgame wisdom via magazines, training DVDs and books (2001, 2008, 2016). Yakov Konoval was the first computer scientist to create a program efficient enough to generate 7-man EGTs in reasonable time. His fruitful partnership with Marc Bourzutschky not only addressed all 7-man endgames but set new standards in the independence of EGT-verification. New depth records were repeatedly set, in this case to the Depth to Conversion metric DTC. New chessic knowledge and insights were also created and many games and studies were highlighted where optimal moves had previously been missed. Having said that, the existence of definitive EGTs benchmarks also highlights the excellence of endgame play at the top level. The 7-man findings of Bourzutschky and Konoval (2006-13) appeared often in the magazine EG and were reviewed in the ICGA Journal (Haworth, 2005-13). A page on the authors themselves would have been an interesting addition.

\footnotetext{
${ }^{1}$ 33, Alexandra Rd., Reading, Berkshire, RG1 5PG, UK. Email: g.haworth@ reading.ac.uk

${ }^{2}$ DTM $\equiv$ Depth to Mate. DTZ $\equiv$ Depth to Zeroing of the move-count. The DTZ 50 metric considers the 50-move-draw rule and indicates possibly-modified depths: frustrated wins, $f w$, are 'draws'. $\mathrm{DTZ}_{50}$ ' also gives depths to frustrated wins. DTZ $_{50}$ ' and therefore DTZ 50 have been calculated for all 6-man endgames whereas DTZ has not.
} 
Table 2

The structure of URE's KRPPKRP analysis.

\begin{tabular}{|c|c|c|c|c|c|c|c|c|c|c|c|}
\hline \multirow[b]{2}{*}{ \# } & \multirow[b]{2}{*}{$\S$} & \multirow[b]{2}{*}{ Class } & \multirow[b]{2}{*}{ Configs. } & \multirow{2}{*}{\multicolumn{2}{|c|}{ pages }} & \multirow{2}{*}{$\begin{array}{c}\text { Games } \\
\text { Total } \\
\end{array}$} & \multicolumn{2}{|c|}{ Draws } & \multicolumn{2}{|c|}{ Wins } & \multirow[b]{2}{*}{ Subsections (P-file configs., ...) } \\
\hline & & & & & & & \# & $\%$ & $\#$ & $\%$ & \\
\hline 01 & 4.3 & $\begin{array}{l}\text { No passed pawns, and } \\
\text { pawns connected }\end{array}$ & 7 & 28 & $67-95$ & 24,007 & 13,144 & 55 & 10,863 & 45 & 7 hg-h/g, fg-g/f, ef-f/e, de-d \\
\hline 02 & 4.4 & $\begin{array}{l}\text { No passed pawns, and } \\
\text { pawns isolated }\end{array}$ & 3 & 4 & $95-99$ & 4,487 & 2,753 & 61 & 1,734 & 39 & 1 fh-g, eg-f etc \\
\hline 03 & 4.5 & $\begin{array}{l}\text { The attacker has a } \\
\text { connected passed pawn }\end{array}$ & 6 & 29 & $99-128$ & 12,358 & 5,478 & 44 & 6,880 & 56 & 6 hg-f, gf-e/h, fe-d/g, ed-f \\
\hline 04 & 4.6 & $\begin{array}{l}\text { The attacker has a nearby, } \\
\text { isolated passed pawn 'apP' }\end{array}$ & 25 & 20 & $128-148$ & 25,510 & 13,962 & 55 & 11,548 & 45 & $6 \mathrm{hf}-\mathrm{h}, \mathrm{eg}-\mathrm{g} / \mathrm{h}, \mathrm{eh}-\mathrm{g} / \mathrm{h}, a p P$ on c-f \\
\hline 05 & 4.7 & One outside passed pawn & 26 & 27 & $148-175$ & 36,677 & 17,017 & 46 & 19,660 & 54 & 15 ah/cg-g/h, bg-f/g/h, af-f/g, .. \\
\hline 06 & 4.8 & $\begin{array}{l}\text { All passed pawns, } \\
\text { connected and close }\end{array}$ & 9 & 5 & $175-180$ & 12,116 & 3,716 & 31 & 8,400 & 69 & 4 Assorted $\mathrm{R} / \mathrm{K} / \mathrm{P}$ properties \\
\hline 07 & 4.9 & $\begin{array}{l}\text { All passed pawns, } \\
\text { connected and far apart }\end{array}$ & 6 & 13 & $180-193$ & 15,788 & 4,781 & 30 & 11,007 & 70 & 5 ab-f/g/h, bc-g/h \\
\hline 08 & 4.10 & $\begin{array}{l}\text { Attacker's pawns passed, } \\
\text { is olated and close together }\end{array}$ & 16 & 4 & 193-197 & 13,383 & 7,288 & 54 & 6,095 & 46 & 3 ac/bd-pP, (e.g.) eh-b \\
\hline 09 & 4.11 & $\begin{array}{l}\text { Attacker's pawns passed, } \\
\text { isolated and far apart }\end{array}$ & 14 & 7 & 197-204 & 13,902 & 7,326 & 53 & 6,576 & 47 & 1 Many cases and motifs \\
\hline 10 & 4.12 & $\begin{array}{l}\text { Doubled pawns versus } \\
\text { pawn }\end{array}$ & 32 & 5 & 204-208 & 5,765 & 3,869 & 67 & 1,896 & 33 & 1 Again, a variety of themes \\
\hline $\mathrm{Tc}$ & & KRPPKRP & 144 & & 142 & 163,993 & 79,334 & 48 & 84,659 & 52 & 49 \\
\hline
\end{tabular}

Some $10 \%$ of games arrive at a rook endgame, and the main focus of this new book is on KRPPKRP to which 160 pages are devoted. The preparatory material necessarily includes KRPKR (14pp), KRPPKR (23pp), KRKP (4pp) and KRPKRP (11pp), and refers to the earlier rook-endgame work of Müller (2001, 2008) and Nunn (1992, 2009, 2010). The last endgames highlighted are KQRPKQR and KRRPKRR (7pp). Each chapter is laced with history, corrections to past analysis, new discoveries and exercises for the reader. Closing out, there are chapters on principles of play, theoretical endgames, historic games and solutions to the exercises. There are comprehensive indexes on players, composers and analysts: all world champions across 130 years from Steinitz to Carlsen have contributed examples of play.

Examples of longest wins are listed as usual and will fascinate those interested in the most profound, subtle and inscrutable play, especially as the initial positions are unlikely to occur on the board. See Tables 1 and 3 here, and Haworth (2017) for extended statistics and lines of play. The practical player will be more assisted by the three tables of statistics, showing the distribution and win/draw profile of actual games across the various parts into which the endgames are divided. KRPKR for example is simply studied in terms of the four files for the pawn: $\mathrm{a} / \mathrm{h}, \mathrm{b} / \mathrm{g}$ etc. The $\mathrm{b} / \mathrm{g}$ files feature the most games $(18,830$ of 60,802$)$ and the highest likelihood of winning (48\%).

KRPKRP is divided according to whether there are passed pawns or not. Winning chances are $31 \%$ on average with maxDTC (per Pp-file arrangement) varying from 25 moves for $\operatorname{KRP}(a) \operatorname{KRP}(b)$ to 41 for $\operatorname{KRP}(a) \operatorname{KRP}(\mathrm{c})$.

Devising a taxonomy for KRPPKRP is the data-mining grand challenge solved by this book. There are 144 configurations of the pawns ${ }^{3}$ and these are discussed under ten themes based mainly on the relative positions of the pawns' files, see Table 2 which is based on the book's p66. Sub-configurations are again

\footnotetext{
${ }^{3}$ Exercise for the reader? Black pawn on files a-d; White pawns on aa/ab/.../hh; $4 * \mathrm{C}(2+7,2)=4 *(9 * 8 / 2)=144$.
} 
usually in terms of specific files for the pawns - ab-a (the most common, $46 \%$ wins), bc-a (63\% wins), gh-a (69\% wins) etc. Even where pawn-files are not used, readers should easily be able to find the relevant part of the KRPPKRP chapter.

Table 3

Some highlighted rook endgame positions, with references to $U R E$.

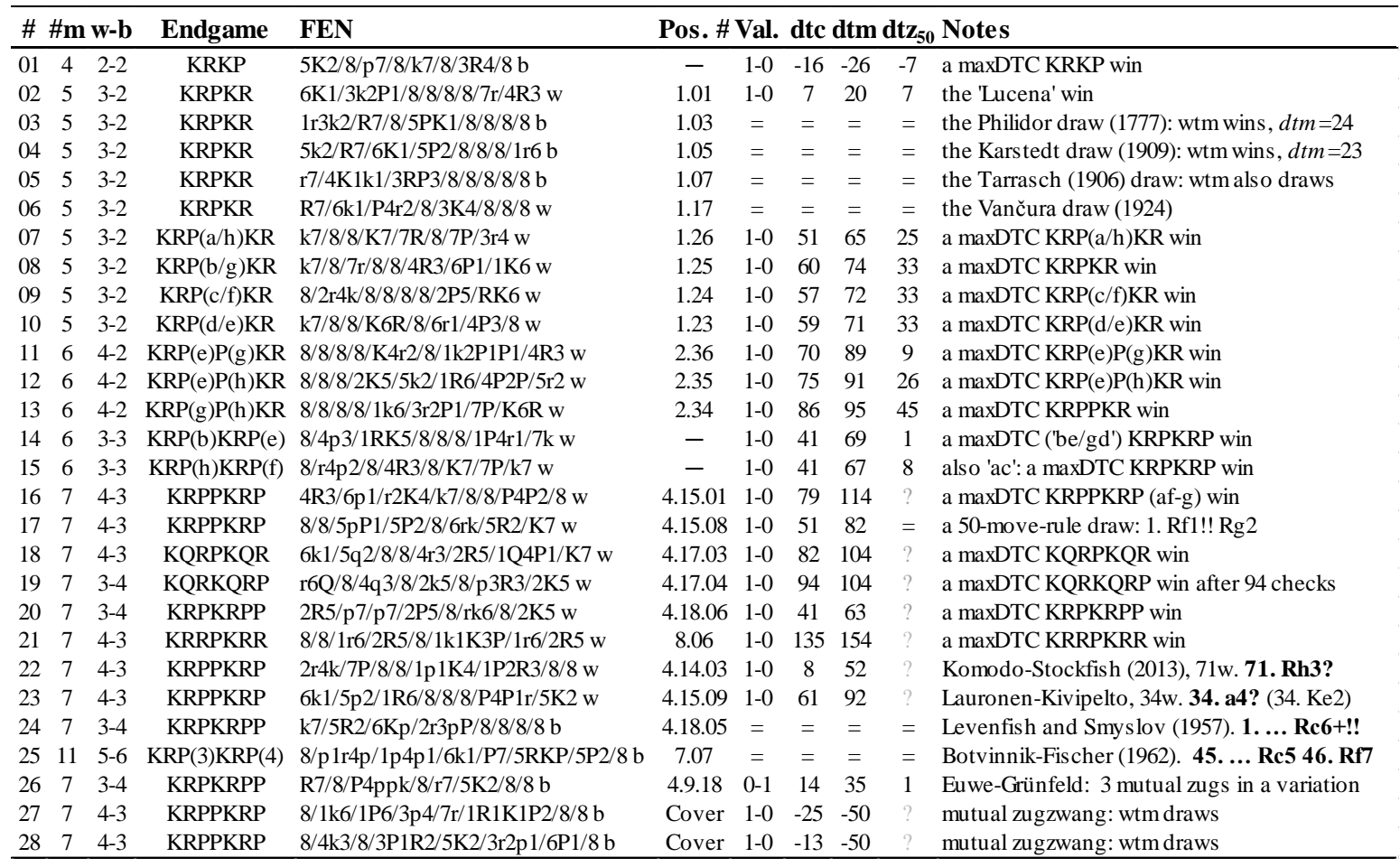

The remainder of this review aims to capture the range and depth of research, and the flavour of the book's text, using just six Table 3 positions (\#02 and \#22-26) as illustrated in Figure 1.

No book on rook endgames is complete without the misnamed KRPKR 'Lucena' position which is traced back (Nunn, 1992, \#179; Winter, 2016) to Salvio (1634) rather than to de Lucena (1497). This shows a common winning technique: the pawn when off the edge allows the king and rook to line up together behind it to exploit the opposing rook's lack of diagonal mobility. "1. Re4. The rook moves up the board to provide a shield for the king. 1. ... Rh1 2. Kf7 Rf1+ 3. Kg6 Rg1+ 4. Kf6 Rf1+ (4. .. Kd6 5. Re6+ Kd7 6. $\operatorname{Re} 5 \operatorname{Rg} 2$ 7. $\operatorname{Rg} 5$ +-) $\mathbf{5} . \mathbf{K g} 5 \mathbf{R g} 1+\mathbf{6} . \mathbf{R g} 4$ +-. This technique is often referred to as 'building a bridge', with White's king and rook forming the pillar in the middle. The Lucena win can also be used with a bishop's or central pawn."

KомODO-STOCKFISH shows that even a top class chess engine can be confounded by a zugzwang. "71. Rh3? (71. Kd5! Rc3 72. Ke4! Rc5 73. Rh3) Rc6 72. Ke4 Rc5 and now White is to move in the mutual zugzwang. 73. Rd3 Kxh7 74. Rd4 Kg7 75 Rxb4 [KRPKR] Kf6 76. Kd4 Rg5 77. Ke4 Ke6 78. Rb5 Rg1 79. Kc5 Kd7 80. Rb7+ Kc8 81. Rb6 and the game was later drawn." There are other examples in the 'computer games' $\$ 4.14$ of engines struggling with fortresses and positional draws. 

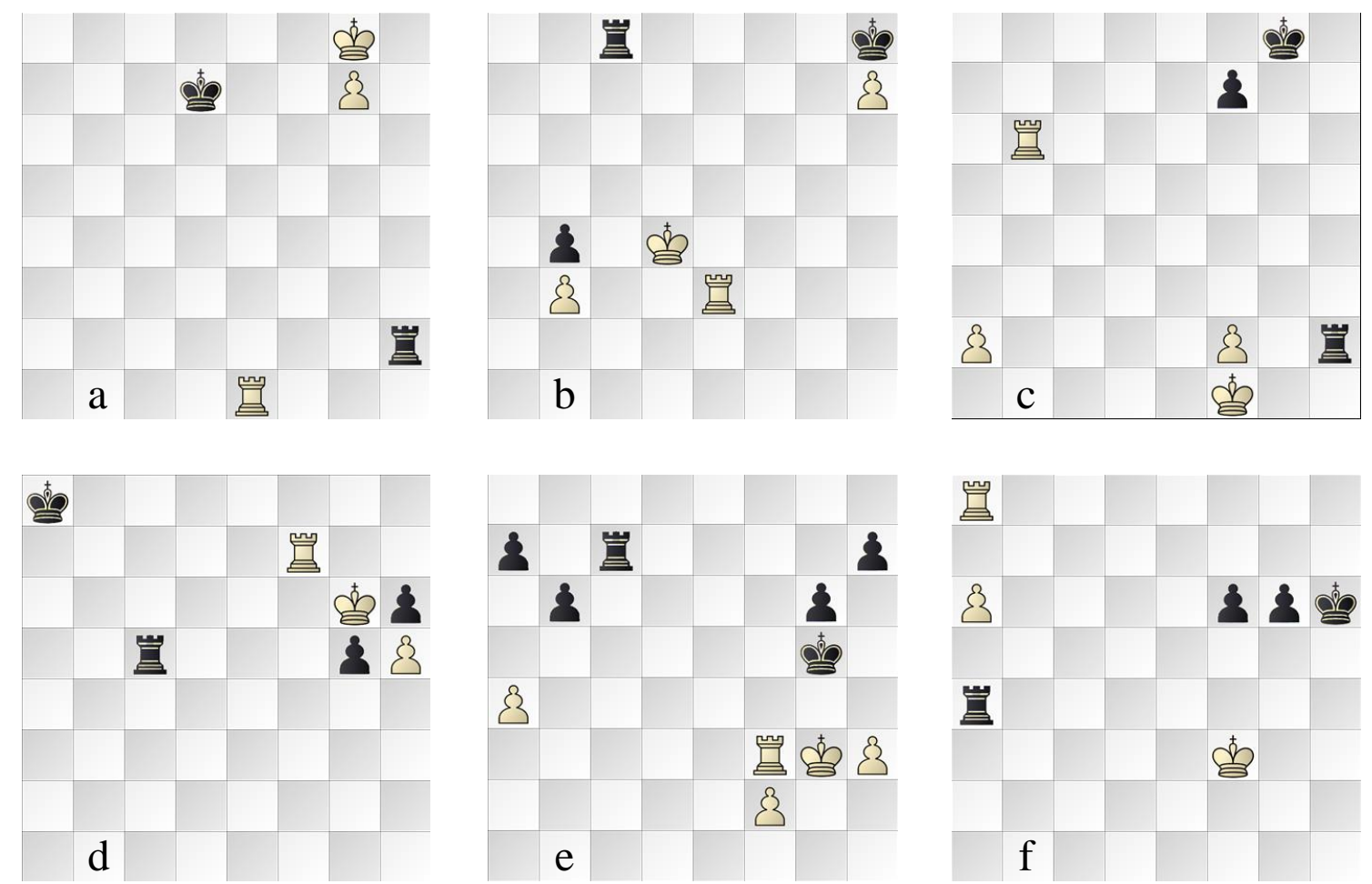

Fig. 1. Table 3 positions: a) \#02 'Lucena', b) \#22 KoмODO-StocKFISH, c) \#23 Lauronen-Kivipelto, d) \#24 Levenfish and Smyslov, e) \#25 Botvinnik-Fischer and f) \#26 Euwe-Grünfeld.

Lauronen-Kivipelto includes the DTC-deepest KRPPKRP position which has occurred over the board: $d t c=61$ compared with the maxDTC of 79 moves. ${ }^{4}$ "34. a4? The win starts with 34 . Ke2! Rh4 35. a3! Kg7 36. Rb2! Ra4 37. Ra2! 34. ... Rh1+? (34. ... Rh5=) 35. Ke2 Ra1 36. Rb4 Ra3 37. f4? (37. Rf4) 37. ... Kg7 38. Kd2 Kg6 39. Kc2 Kf5 40. Kb2 Rh3 41. a5 Kg4 42. a6 Rh6 43. Ra4 Rh8 44. Kc3 f5 45. a7 Ra8 46. Kd3 Kf3 47. Kd2 Rd8+ 48. Ke1 Re8+ 49. Kf1 Ra8 50. Kg1 Rg8+ 51. Kf1 Ra8 52. Ke1 Re8+ 53. Kd2 Rd8+ 54. Kc3 Ra8 55. Kd3 Kg4? (55. ... Kg3=) 56. Ke3 Re8+ 57. Kf2 Ra8 5s8. Kg2 Kh4 59. Kf3 Kh5 60. Ra6 1-0."

The position from Levenfish and Smyslov (1986, 1989), originally published in 1957, has already been analysed by Nunn (2014) in his EGT-assisted revisit of that book. The URE text: "According to Levenfish and Smyslov, White wins, but Black has a nice draw: 1.... Rc6+ (1...g4? 2. Kxh6 g3 (2... Rc6+ 3. Kh7 Kb8 4. Rf4 g3 5. Rg4 Rc3 6. Kg6 +-) 3. Rg7 Rc3 4. Rg4 Ka7 5. Kg5 +-) 2. Kh7 Kb8 3. Rg7 Kc8? This move loses as does 3. ... Rc7? 4. Kxh6 Rxg7 5. Kxg7 g4 6. h6 +-. Black can hold by 3. ... g4 4. Rxg4 Kc7 5. Rg6 Rc5!! 6. Kxh6 Kd7! = or 3. ... Rd6 4. Rg6 Kc7 5. Kxh6 g4 6. Kh7 Rd7+ 7. Kg8 Rd8+ 8. Kf7 Rd5!! (Levenfish and Smyslov missed this tactical nuance) 9. h6 Rd7+ 10. Ke6 Rd6+ 11. Kf5 Rxg6 and White doesn't have hxg6. The move 8. ... Rd5!! was also found by John Nunn. 4. Rg6 Kb7 5. Kxh6 g4 6. Kh7 Rc7+ 7. Kg8 Rc8+ 8. Kf7 Rc5 9. h6 Rc7+ 10. Kg8 Rc8+ 11. Kh7 Rc7+ 12. Rg7 g3 13. Kh8! +-."

From the Historic Games chapter, the only Botvinnik-Fischer confrontation. "One of the most famous adjourned games. Fischer had sealed 45. ... Rc5. 46. Rf7 Ra5 47. Rxh7. The Soviet team had analysed all

\footnotetext{
${ }^{4}$ \#8.05, Dyckhoff-Eliskases (1930), 0-1: KRRKRRP, 4R3/3r4/8/8/2K1p2R/5k2/4r3/8 b - 12 69, dtc = 79 moves. The 50-move rule might have intervened with best defence but White resigned on move 86.
} 
night long and established that Geller's fantastic idea was sufficient for a draw. This concept of fighting against Black's queenside pawns had escaped Fischer's attention. 47. ... Rxa4 48. h4+ Kf5 49. Rf7+ Ke5 50. Rg7 Ra1 51. Kf3 b5?! After 51... Kd4 52. Rxg6 b5 53. h5 b4 54. h6 b3 55. Rg4+ Kc5 56. Rg5+ Kc6 57. Rg6+ Kb7 58. Rg7+ Ka6 59. Rg6+ Ka5 60. Rg5+ Ka4 61. Rg4+ Ka3 62. Rh4 b2 63. h7 b1=Q 64. h8=Q Qb3+ 65. Ke2 Qd1+ 66. Ke3 Rb1 Fischer claimed that Black wins in My 60 Memorable Games. But Botvinnik found that 67. Qf8+ Ka2 68. Qc5 draws and the 13-year-old Garry Kasparov even found a second drawing method in a session of the Botvinnik school: 67. Rc4! =. 52. h5! Ra3+ 53. Kg2 gxh5 54. Rg5+ Kd6 55. Rxb5 [KRPKRPP, =] h4 56. f4 Kc6 57. Rb8 h3+ 58. Kh2 a5 59. f5 Kc7 60. Rb5 Kd6 61. f6 Ke6 62. Rb6+ Kf7 63. Ra6 Kg6 64. Rc6 a4 65. Ra6 Kf7 66. Rc6 Rd3 67. Ra6 a3 68. Kg1 'With a face as white as a sheet, Fischer shook my hand and left the hall with tears in his eyes' (Botvinnik)."

Euwe-Grünfeld (1921) features three mutual zugzwangs in a variation just off the played line. "This is a wellknown theoretical position. 48.... Kg5? Black's king should not move in front of the pawns. One sample win is 48. ... f5 49. a7 Kg7 50. $\mathrm{Kg} 3$ g5 51. Kf3 Ra3+ 52. Kg2 g4 53. Kf2 f4 54. Rb8 Rxa7 55. Rb4 Ra2+ 56. Kg1 $\mathrm{Ra} 1+57 . \mathrm{Kf} 2 \mathrm{~g} 3+58 . \mathrm{Kg} 2 \mathrm{Ra} 2+59 . \mathrm{Kg} 1 \mathrm{Rf} 2+-.49$. a7 Kf5 50. Kg3? This curious error also occurs in Dvoretsky's Endgame Manual (though corrected in the $4^{\text {th }}$ edition of 2014), Levenfish and Smyslov's famous rook endings book and several practical games. The correct line is $50 . \mathrm{Kg} 2 \mathrm{Ra} 2+51 . \mathrm{Kg} 3 \mathrm{~g} 552 . \mathrm{Kf} 3 \mathrm{Ra} 3+$ 53. $\mathrm{Kg} 2 \mathrm{Kg} 4$ 54. Rf8 Ra2+ 55. $\mathrm{Kg} 1 \mathrm{Rxa} 7$ 56. Rxf6 Kg3 57. Rf1 =. 50. ... Ra3+? After 50. ... Ra2! with mutual zugzwang, Black wins, e.g., 51. Kf3 g5 mz 52. Kg3 (52. Ke3 g4 mz) 52. ... g4 53. Rb8 Ra3+ 54. Kf2 Rxa7 + . 51. Kf2 Ra2+ 52. Kg3 Kg5 53. Kf3 f5 54. Kg3 Ra3+ 55. Kg2 f4 56. Kf2 Kg4 57. Rg8 Ra2+ 58. Kg1 Rxa7 59. Rxg6+ Kf3 60. Rf6 Ra1+ 61. Kh2 Re1 62. Rf8 Re4 63. Kg1 Re1+ 64. Kh2 Rf1 65. Ra8 $1 / 2-1 / 2 . "$

Players at all levels will benefit from URE's new approach and insights into rook endgames, particularly KRPPKRP. There are many mansions in the world of chess: the game, the study, the puzzle, the statistics and the historical heritage. Whatever one's interest, there is much to discover, savour, absorb and enjoy here. The Gambit team's professional editorial and production standards are well in evidence with an intriguing 'deus ex machina' cover, balanced coverage edited by Graham Burgess, typesetting by Petra Nunn and a perceptive foreword from John Nunn with his own choice of highlights. Understanding Rook Endgames is a major and definitive step forward on the topic, sets the bar high and will surely be the reference text for years to come. It entertains as much as it educates and is thoroughly welcome and recommended from all points of view.

My thanks to Yakov Konoval for tabular data which could not be accommodated in URE, to colleagues for supplementary information, and to Eiko Bleicher (2017), Ronald de Man and Niklas Feikas (2017), John Tamplin (2017) and Victor Zakharov (2017) for the service provided by their DTC, DTM and DTZ50' EGTquery sites.

\section{REFERENCES}

Bleicher, E. (2017). http://www.k4it.de. EGT-query service on Nalimov-style sub-6-man DTC/Z EGTs.

Bourzutschky, M. and Konoval, Y. (2006-13) Findings from 7-man DTC EGTs. EG issues 11.162, 17.185/6, 18.188/190 and 19.191.

De Lucena, L. R. (1497). Repetición de Amores y Arte de Ajedrez con 101 Juegos de Partido. Online copy at http://preview.tinyurl.com/hojen5v.

Dvoretsky, M. (2003). Dvoretsky's Endgame Manual. Russell Enterprises. $4^{\text {th }}$ edition, 2014. 
Fiekas, N. (2017). https://syzygy-tables.info/. Query service on de Man's 'syzygy' DTZ50' EGTs.

Haworth, G. M ${ }^{\mathrm{c} C}$. (2005-13) Reviews of MB/YK 'EG' articles on 7-man DTC EGT discoveries. ICGA Journal issues 28.4, 32.4, 34.2, 35.2, 36.1 and 36.2.

Haworth, G. $M^{c} C$. (2017). http://centaur.reading.ac.uk/65694/. This review plus supplementary data and pgn file.

Левенфиш, Г., Смыслов В. (1986). Теория ладейных окончаний, (The Theory of Rook Endings), $3^{\text {rd }}$ revised and extended edition. YoYo Media, Moscow.

Levenfish G. and Smyslov, V. (1989). Rook Endings. 2nd revised edition. Batsford. ISBN 9780713458091.

Müller, K. (2014). Chess Endgames. DVD series. Chessbase.

Müller, K. and Lamprecht, F. (2001). Fundamental Chess Endings, Gambit, esp. chapter 6.

Müller, K. and Konoval, Y. (2016). Understanding Rook Endgames. Gambit. ISBN-13 978191-0093818.

Müller, K. and Pajeken, W. (2008). How to Play Chess Endgames. Gambit. ISBN-13: 978190-4600862.

MVL team (2017). http://tb7.chessok.com/probe. DTM-evaluation service for sub-8-man endgames.

Nunn, J. (1992). Secrets of Rook Endings. Gambit. Second edition (1999). ISBN-13: 978190-1983180.

Nunn, J. (2009). Understanding Chess Endgames, Gambit. ISBN-13: 978190-6454111.

Nunn, J. (2010). Nunn's Chess Endings Volume 2, Gambit, esp. chapter 8. ISBN-13: 978190-6454234.

Nunn, J. (2014). Discoveries in R+2P v R+P Endings. ICGA Journal, Vol. 36, No. 3, pp. 139-142.

Salvio, A. (1634). Il Puttino. Online copy at http://preview.tinyurl.com/h8ckttg.

Tamplin, J. (2017). http://chess.jaet.org/endings/ DTC/Z/Z50 EGT-query service.

Winter, E. (2014). The Lucena Position. http://www.chesshistory.com/winter/extra/lucena.html.

Zakharov, V. (2016). http://tb7.chessok.com/. The 'MVL' query-service on the Lomonosov sub-8-man DTM EGTs. 